\title{
Science over speed
}

\begin{abstract}
President Donald Trump's call to speed up drug approval by the US Food and Drug Administration (FDA) overlooks the fact that the agency has become faster over the past two decades. When considering changes to the drug-approval process, we should instead increase our investment in the many years of research that precede approval.
\end{abstract}

$\mathbf{E}$ arlier this year, at his first address to a joint session of the US Congress, President Donald Trump criticized the drug regulatoryapproval process in the US, calling it "slow and burdensome." As this issue went to press, he had recently nominated physician Scott Gottlieb as the next FDA commissioner. In comparison to short-listed candidate Jim O'Neill, a venture capitalist who has suggested that drug efficacy need only be proven after a drug has been approved, Gottlieb seems to be a safer choice. But Gottlieb, too, has described in past the FDA's "excessive desire for certainty" - a quality that, according to him, stems from a mistrust of the companies that market FDA-regulated medications and of the doctors who ultimately prescribe these drugs (see go.nature.com/2moshpc). He has also called the FDA's approval process "cumbersome."

Yet data suggest that the FDA's regulatory process might be the fastest it has ever been. December 2016 figures show that the agency, on average, approved drugs within eight months of submission last year (see go.nature.com/2ngf3dt). This timeframe has been steadily decreasing for more than two decades, from a period of over 19 months in 1993 to close to 11 months in 2015. Moreover, some evidence suggests that the FDA may outpace other major regulatory bodies around the world. A 2015 analysis by the Centre for Innovation in Regulatory Science compared the approval timelines of the FDA, European Medicines Agency, Health Canada, Swissmedic, Australia’s Therapeutic Goods Administration and Japan's Pharmaceuticals and Medical Devices Agency (PMDA), and found that, for most of the decade from 2006 to 2015, the FDA consistently held the title for fastest drug approvals. It was only in 2014 and 2015 that the PMDA moved slightly ahead of the FDA. In 2015, the PMDA approved drugs roughly 284 days after submission as compared to the FDA's 351 days (see go.nature.com $/ 2 \mathrm{mx} 00 \mathrm{hh}$ ).

Even if the FDA were to expedite its regulatory review, it's unclear whether 'faster' translates to a better system. Currently, the FDA coordinates with scientific experts, including clinicians, nonphysician researchers, statisticians and patients, to evaluate products submitted for review. Accelerating assessments of safety or efficacy, or even reducing the number of stakeholders involved, could prove risky. At least one study has found that faster drug approvals have led to a higher rate of drugs being withdrawn or needing black-box warnings after approval (Health Aff. 33, 1453-1459, 2014).

The prospect of a sped-up drug-approval system is also concerning given that we already have reason to worry about compromised standards. The $21^{\text {st }}$ Century Cures Act was signed into law in December
2016 and gives the FDA more funding to hire scientific experts. But, as a previous editorial has pointed out, the Act allows the FDA to set up a framework to incorporate adapted clinical trial designs, including using surrogate markers, shorter trial times and a smaller study population, into its approval process (Nat. Med. 23, 1, 2017). Some worry that this allowance might incentivize the agency to rely increasingly on these alternate data and trial designs. But the FDA has already been doing this, it turns out-especially for accelerated approvals and orphan drugs. A 2014 study looked at novel agents approved by the FDA between 2005 and 2012 and found that roughly 74 of the 206 indications for these new drugs were approved on the basis of a single pivotal trial. Furthermore, 91 indications were approved primarily using surrogate endpoints that were expected to demonstrate clinical benefit, regardless of actual benefit (JAMA 311, 368-377, 2014). Many in the biopharma industry do not think that the FDA's approval period needs speeding up. They have also expressed concern that an accelerated regulatory process could lead to less robust reviews of drugs, and in turn, a lower chance of insurers providing reimbursement for new medicines.

What's more, the bottleneck in bringing new drugs to market might not be the approval process, but rather, the drug-discovery and development pipeline. Some industry groups estimate that it takes between 10 and 15 years for a drug to go from initial discovery to the marketplace. Even so, the chances that a drug makes it from clinical testing to market are estimated in some cases to be less than $12 \%$ (see go.nature. com/2lxo7p5). The focus should instead be on the years of preclinical research before a drug enters trials. Related to this, on $16 \mathrm{March}$, the White House released its budget proposal for the upcoming fiscal year, including a near-\$6-billion reduction in funding allotted to the US National Institutes of Health (NIH). President Trump and others calling for deregulation at the FDA should know that many drugs get their start in basic science, a field largely funded by the NIH. Such decimation of the agency's budget is likely to hinder innovation, and ultimately, leave patients with fewer new options.

There is no disagreement on the part of lawmakers, pharmaceutical companies and patients alike that getting drugs to market sooner would be desirable. President Trump has thus far not been explicit about how, beyond the time to market, the drug-approval process needs to change. But with most approvals taking between six months and a year, as well as a move toward lowered benchmarks, the administration would do better to shift its focus to the basic scientific development on which approval depends. 